Network Working Group

Request for Comments: 3471

Category: Standards Track
L. Berger, Editor

Movaz Networks

January 2003

\title{
Generalized Multi-Protocol Label Switching (GMPLS)
}

Signaling Functional Description

Status of this Memo

This document specifies an Internet standards track protocol for the Internet community, and requests discussion and suggestions for improvements. Please refer to the current edition of the "Internet Official Protocol standards" (STD 1) for the standardization state and status of this protocol. Distribution of this memo is unlimited.

Copyright Notice

Copyright (C) The Internet Society (2003). All Rights Reserved.

Abstract

This document describes extensions to Multi-Protocol Label Switching (MPLS) signaling required to support Generalized MPLS. Generalized MPLS extends the MPLS control plane to encompass time-division (e.g., Synchronous optical Network and Synchronous Digital Hierarchy, SONET/SDH), wavelength (optical lambdas) and spatial switching (e.g., incoming port or fiber to outgoing port or fiber). This document presents a functional description of the extensions. Protocol specific formats and mechanisms, and technology specific details are specified in separate documents.

Table of Contents





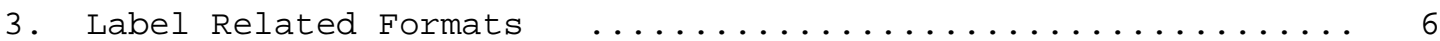















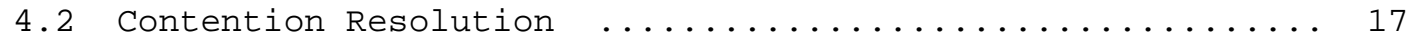

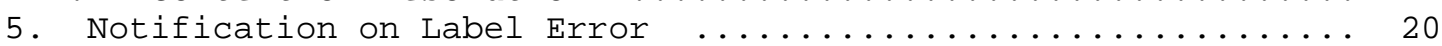










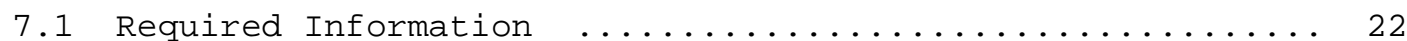









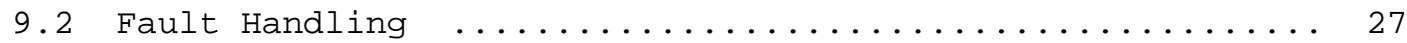







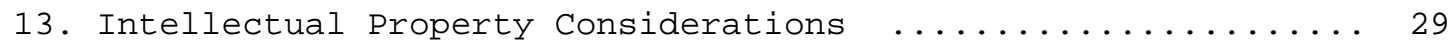

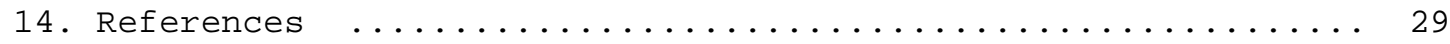

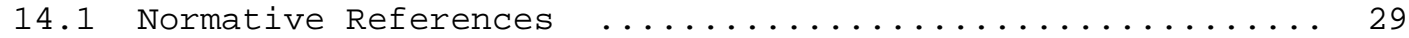

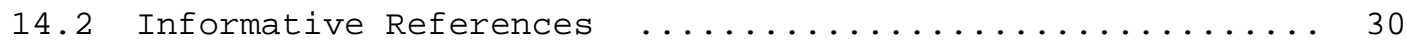

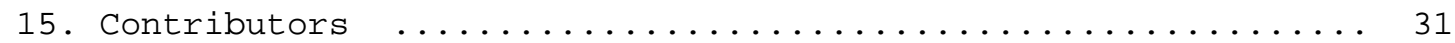

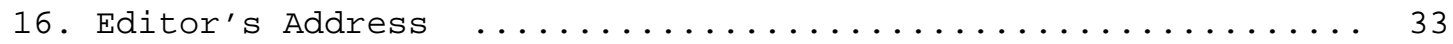

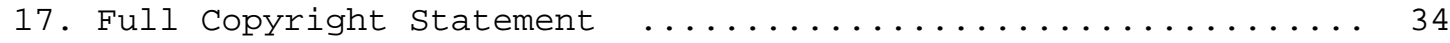

1. Introduction

The Multiprotocol Label Switching (MPLS) architecture [RFC3031] has been defined to support the forwarding of data based on a label. In this architecture, Label switching Routers (LSRs) were assumed to have a forwarding plane that is capable of (a) recognizing either packet or cell boundaries, and (b) being able to process either packet headers (for LSRs capable of recognizing packet boundaries) or cell headers (for LSRs capable of recognizing cell boundaries).

The original architecture has recently been extended to include LSRS whose forwarding plane recognizes neither packet, nor cell boundaries, and therefore, can't forward data based on the information carried in either packet or cell headers. Specifically, such LSRs include devices where the forwarding decision is based on time slots, wavelengths, or physical ports.

Given the above, LSRs, or more precisely interfaces on LSRs, can be subdivided into the following classes:

1. Interfaces that recognize packet/cell boundaries and can forward data based on the content of the packet/cell header. Examples include interfaces on routers that forward data based on the content of the "shim" header, interfaces on (Asynchronous Transfer Mode) ATM-LSRs that forward data based on the ATM VPI/VCI. Such interfaces are referred to as Packet-Switch Capable (PSC). 
2. Interfaces that forward data based on the data's time slot in a repeating cycle. An example of such an interface is an interface on a SONET/SDH Cross-Connect. Such interfaces are referred to as Time-Division Multiplex Capable (TDM) .

3. Interfaces that forward data based on the wavelength on which the data is received. An example of such an interface is an interface on an Optical Cross-Connect that can operate at the level of an individual wavelength. Such interfaces are referred to as Lambda Switch Capable (LSC).

4. Interfaces that forward data based on a position of the data in the real world physical spaces. An example of such an interface is an interface on an Optical Cross-Connect that can operate at the level of a single (or multiple) fibers. Such interfaces are referred to as Fiber-Switch Capable (FSC).

Using the concept of nested Label Switched Paths (LSPs) allows the system to scale by building a forwarding hierarchy. At the top of this hierarchy are FSC interfaces, followed by LSC interfaces, followed by TDM interfaces, followed by PSC interfaces. This way, an LSP that starts and ends on a PSC interface can be nested (together with other LSPS) into an LSP that starts and ends on a TDM interface. This LSP, in turn, can be nested (together with other LSPs) into an LSP that starts and ends on an LSC interface, which in turn can be nested (together with other LSPS) into an LSP that starts and ends on a FSC interface. See [MPLS-HIERARCHY] for more information on LSP hierarchies.

The establishment of LSPs that span only the first class of interfaces is defined in [RFC3036, RFC3212, RFC3209]. This document presents a functional description of the extensions needed to generalize the MPLS control plane to support each of the four classes of interfaces. Only signaling protocol independent formats and definitions are provided in this document. Protocol specific formats are defined in [RFC3473] and [RFC3472]. Technology specific details are outside the scope of this document and will be specified in technology specific documents, such as [GMPLS-SONET].

The key words "MUST", "MUST NOT", "REQUIRED", "SHALL", "SHALL NOT", "SHOULD", "SHOULD NOT", "RECOMMENDED", "MAY", and "OPTIONAL" in this document are to be interpreted as described in [RFC2119].

2. Overview

Generalized MPLS differs from traditional MPLS in that it supports multiple types of switching, i.e., the addition of support for TDM, lambda, and fiber (port) switching. The support for the additional 
types of switching has driven generalized MPLS to extend certain base functions of traditional MPLS and, in some cases, to add functionality. These changes and additions impact basic LSP properties, how labels are requested and communicated, the unidirectional nature of LSPs, how errors are propagated, and information provided for synchronizing the ingress and egress.

In traditional MPLS Traffic Engineering, links traversed by an LSP can include an intermix of links with heterogeneous label encodings. For example, an LSP may span links between routers, links between routers and ATM-LSRs, and links between ATM-LSRs. Generalized MPLS extends this by including links where the label is encoded as a time slot, or a wavelength, or a position in the real world physical space. Just like with traditional MPLS TE, where not all LSRs are capable of recognizing (IP) packet boundaries (e.g., an ATM-LSR) in their forwarding plane, generalized MPLS includes support for LSRS that can't recognize (IP) packet boundaries in their forwarding plane. In traditional MPLS TE an LSP that carries IP has to start and end on a router. Generalized MPLS extends this by requiring an LSP to start and end on similar type of LSRs. Also, in generalized MPLS the type of a payload that can be carried by an LSP is extended to allow such payloads as SONET/SDH, or 1 or $10 \mathrm{~Gb}$ Ethernet. These changes from traditional MPLS are reflected in how labels are requested and communicated in generalized MPLS, see Sections 3.1 and 3.2. A special case of Lambda switching, called Waveband switching is also described in section 3.3 .

Another basic difference between traditional and non-PSC types of generalized MPLS LSPS, is that bandwidth allocation for an LSP can be performed only in discrete units, see Section 3.1.3. There are also likely to be (much) fewer labels on non-PSC links than on PSC links. Note that the use of Forwarding Adjacencies (FA), see [MPLSHIERARCHY], provides a mechanism that may improve bandwidth utilization, when bandwidth allocation can be performed only in discrete units, as well as a mechanism to aggregate forwarding state, thus allowing the number of required labels to be reduced.

Generalized MPLS allows for a label to be suggested by an upstream node, see Section 3.4. This suggestion may be overridden by a downstream node but, in some cases, at the cost of higher LSP setup time. The suggested label is valuable when establishing LSPs through certain kinds of optical equipment where there may be a lengthy (in electrical terms) delay in configuring the switching fabric. For example micro mirrors may have to be elevated or moved, and this physical motion and subsequent damping takes time. If the labels and hence switching fabric are configured in the reverse direction (the 
norm) the MAPPING/Resv message may need to be delayed by $10^{\prime} \mathrm{s}$ of milliseconds per hop in order to establish a usable forwarding path. The suggested label is also valuable when recovering from nodal faults.

Generalized MPLS extends on the notion of restricting the range of labels that may be selected by a downstream node, see section 3.5. In generalized MPLS, an ingress or other upstream node may restrict the labels that may be used by an LSP along either a single hop or along the whole LSP path. This feature is driven from the optical domain where there are cases where wavelengths used by the path must be restricted either to a small subset of possible wavelengths, or to one specific wavelength. This requirement occurs because some equipment may only be able to generate a small set of the wavelengths that intermediate equipment may be able to switch, or because intermediate equipment may not be able to switch a wavelength at all, being only able to redirect it to a different fiber.

While traditional traffic engineered MPLS (and even LDP) are unidirectional, generalized MPLS supports the establishment of bidirectional LSPs, see Section 4. The need for bidirectional LSPS comes from non-PSC applications. There are multiple reasons why such LSPs are needed, particularly possible resource contention when allocating reciprocal LSPs via separate signaling sessions, and simplifying failure restoration procedures in the non-PSC case. Bidirectional LSPs also have the benefit of lower setup latency and lower number of messages required during setup.

Generalized MPLS supports the communication of a specific label to use on a specific interface, see section 6. [RFC3473] also supports an RSVP specific mechanism for rapid failure notification.

Generalized MPLS formalizes possible separation of control and data channels, see Section 9. Such support is particularly important to support technologies where control traffic cannot be sent in-band with the data traffic.

Generalized MPLS also allows for the inclusion of technology specific parameters in signaling. The intent is for all technology specific parameters to be carried, when using RSVP, in the SENDER_TSPEC and other related objects, and when using CR-LDP, in the Traffic Parameters TLV. Technology specific formats will be defined on an as needed basis. For an example definition, see [GMPLS-SONET] . 


\section{Label Related Formats}

To deal with the widening scope of MPLS into the optical and time domain, several new forms of "label" are required. These new forms of label are collectively referred to as a "generalized label". A generalized label contains enough information to allow the receiving node to program its cross connect, regardless of the type of this cross connect, such that the ingress segments of the path are properly joined. This section defines a generalized label request, a generalized label, support for waveband switching, suggested label and label sets.

Note that since the nodes sending and receiving the new form of label know what kinds of link they are using, the generalized label does not contain a type field, instead the nodes are expected to know from context what type of label to expect.

\subsection{Generalized Label Request}

The Generalized Label Request supports communication of characteristics required to support the LSP being requested. These characteristics include LSP encoding and LSP payload. Note that these characteristics may be used by transit nodes, e.g., to support penultimate hop popping.

The Generalized Label Request carries an LSP encoding parameter, called LSP Encoding Type. This parameter indicates the encoding type, e.g., SONET/SDH/GigE etc., that will be used with the data associated with the LSP. The LSP Encoding Type represents the nature of the LSP, and not the nature of the links that the LSP traverses. A link may support a set of encoding formats, where support means that a link is able to carry and switch a signal of one or more of these encoding formats depending on the resource availability and capacity of the link. For example, consider an LSP signaled with "lambda" encoding. It is expected that such an LSP would be supported with no electrical conversion and no knowledge of the modulation and speed by the transit nodes. Other formats normally require framing knowledge, and field parameters are broken into the framing type and speed as shown below.

The Generalized Label Request also indicates the type of switching that is being requested on a link. This field normally is consistent across all links of an LSP. 


\subsubsection{Required Information}

The information carried in a Generalized Label Request is:

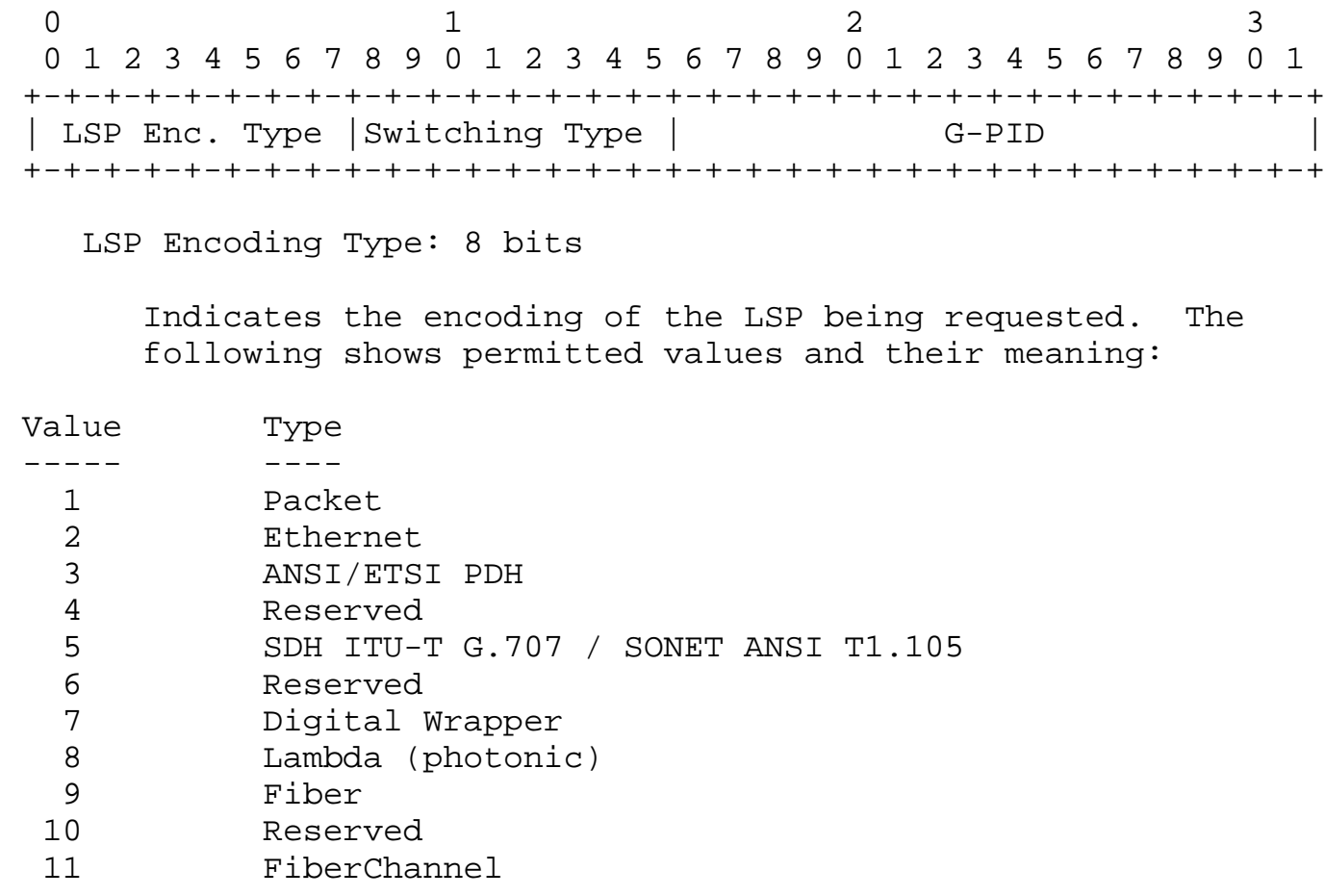




\section{Switching Type: 8 bits}

Indicates the type of switching that should be performed on a particular link. This field is needed for links that advertise more than one type of switching capability. This field should map to one of the values advertised for the corresponding link in the routing Switching Capability Descriptor, see [GMPLSRTG] •

The following are currently defined values:

\begin{tabular}{|c|c|c|}
\hline Value & Type & \\
\hline----- & ---- & \\
\hline 1 & Packet-Switch Capable-1 & $(\mathrm{PSC}-1)$ \\
\hline 2 & Packet-Switch Capable-2 & $(\mathrm{PSC}-2)$ \\
\hline 3 & Packet-Switch Capable-3 & $(\mathrm{PSC}-3)$ \\
\hline 4 & Packet-Switch Capable-4 & $(\mathrm{PSC}-4)$ \\
\hline 51 & Layer-2 Switch Capable & $(\mathrm{L} 2 \mathrm{SC})$ \\
\hline 100 & Time-Division-Multiplex & Capable (TDM) \\
\hline 150 & Lambda-Switch Capable & $(\mathrm{LSC})$ \\
\hline 200 & Fiber-Switch Capable & $(\mathrm{FSC})$ \\
\hline
\end{tabular}




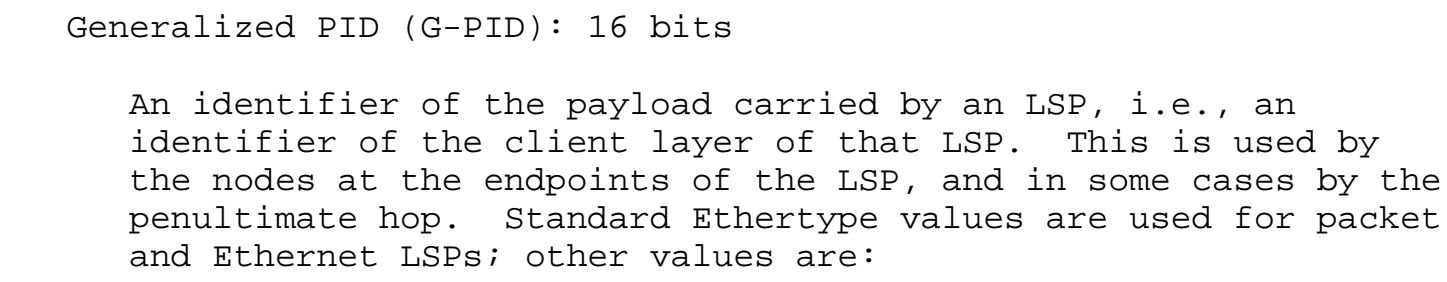

\begin{tabular}{|c|c|}
\hline Value & Type \\
\hline---- & ---- \\
\hline 0 & Unknown \\
\hline 1 & Reserved \\
\hline 2 & Reserved \\
\hline 3 & Reserved \\
\hline 4 & Reserved \\
\hline 5 & Asynchronous mapping of $\mathrm{E} 4$ \\
\hline 6 & Asynchronous mapping of $\mathrm{DS} 3 / \mathrm{T} 3$ \\
\hline 7 & Asynchronous mapping of E3 \\
\hline 8 & Bit synchronous mapping of $\mathrm{E} 3$ \\
\hline 9 & Byte synchronous mapping of $\mathrm{E} 3$ \\
\hline 10 & Asynchronous mapping of DS2/T2 \\
\hline 11 & Bit synchronous mapping of $\mathrm{DS} 2 / \mathrm{T} 2$ \\
\hline 12 & Reserved \\
\hline 13 & Asynchronous mapping of $\mathrm{E} 1$ \\
\hline 14 & Byte synchronous mapping of $\mathrm{E} 1$ \\
\hline 15 & Byte synchronous mapping of 31 * DS0 \\
\hline 16 & Asynchronous mapping of DS1/T1 \\
\hline 17 & Bit synchronous mapping of $\mathrm{DS} 1 / \mathrm{T} 1$ \\
\hline 18 & Byte synchronous mapping of DS1/T1 \\
\hline 19 & $\mathrm{VC}-11$ in $\mathrm{VC}-12$ \\
\hline 20 & Reserved \\
\hline 21 & Reserved \\
\hline 22 & DS1 SF Asynchronous \\
\hline 23 & DS1 ESF Asynchronous \\
\hline 24 & DS3 M23 Asynchronous \\
\hline 25 & Ds3 C-Bit Parity Asynchronous \\
\hline 26 & VT/LOVC \\
\hline 27 & STS SPE/HOVC \\
\hline 28 & POS - No Scrambling, 16 bit CRC \\
\hline 29 & POS - No Scrambling, 32 bit CRC \\
\hline 30 & POS - Scrambling, 16 bit CRC \\
\hline 31 & POS - Scrambling, 32 bit CRC \\
\hline 32 & ATM mapping \\
\hline 33 & Ethernet \\
\hline 34 & SONET/SDH \\
\hline 35 & Reserved (SONET deprecated) \\
\hline 36 & Digital Wrapper \\
\hline 37 & Lambda \\
\hline
\end{tabular}

Technology

All

SDH
SDH
SDH
SDH
SDH
SDH
SDH
SDH
SDH
SDH
SDH
SDH
SDH
SDH

SONET
SONET
SONET
SONET
SDH
SDH
SDH
SDH
SDH
SDH
SDH
SDH, Lambda, Fiber
Lambda, Fiber
Lambda, Fiber
Lambda, Fiber
Fiber




$\begin{array}{lll}38 & \text { ANSI/ETSI PDH } & \text { SDH } \\ 39 & \text { Reserved } & \text { SDH } \\ 40 & \text { Link Access Protocol SDH } & \text { SDH } \\ & \text { (LAPS }- \text { X.85 and X.86) } & \\ 41 & \text { FDD } & \text { SDH, Lambda, Fiber } \\ 42 & \text { DQDB (ETSI ETS } 300216) & \text { SDH } \\ 43 & \text { FiberChannel-3 (Services) } & \text { FiberChannel } \\ 44 & \text { HDLC } & \text { SDH } \\ 45 & \text { Ethernet V2/DIX (only) } & \text { SDH, Lambda, Fiber } \\ 46 & \text { Ethernet } 802.3 \text { (only) } & \text { SDH, Lambda, Fiber }\end{array}$

3.1.2. Bandwidth Encoding

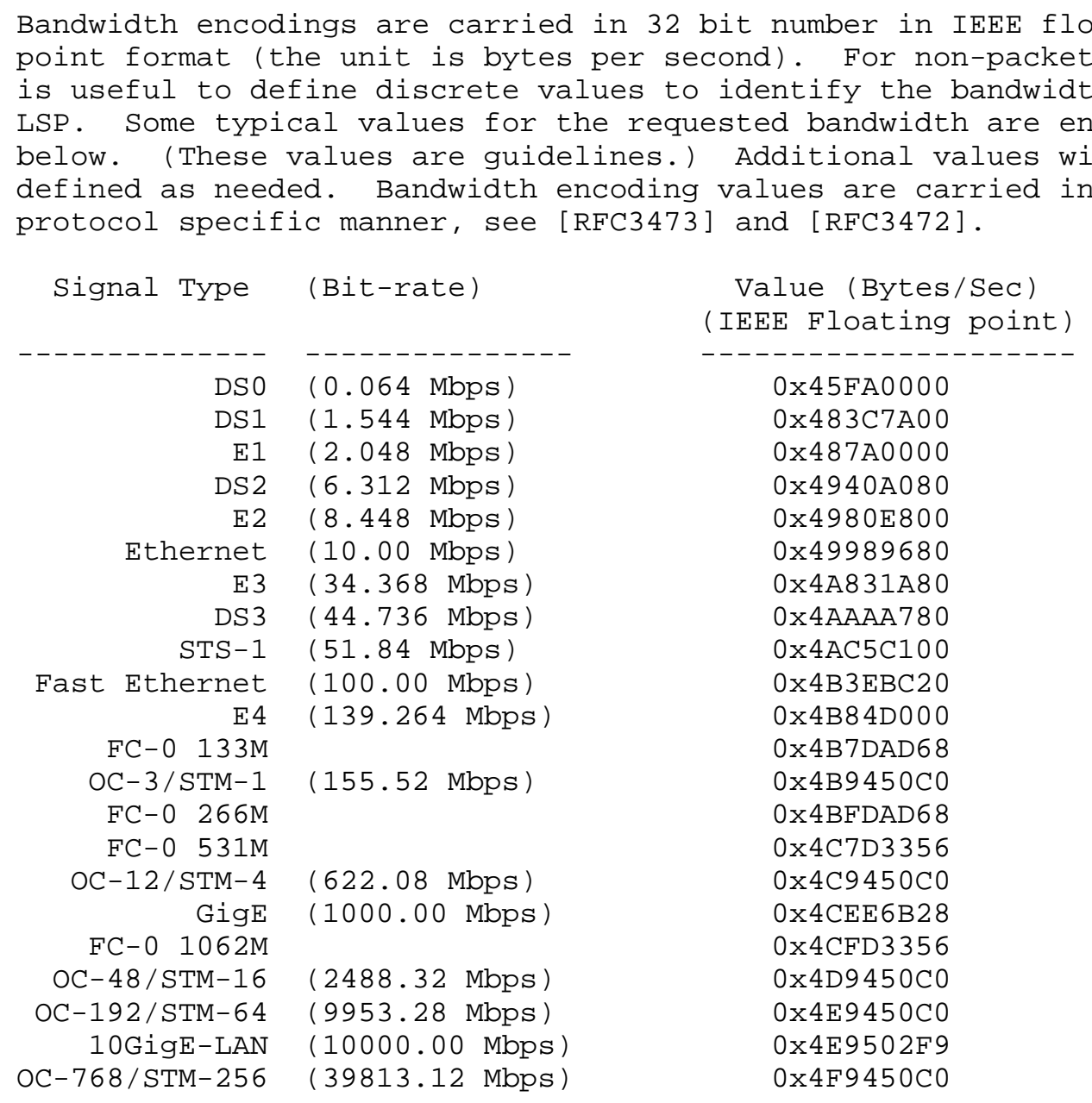




\subsection{Generalized Label}

The Generalized Label extends the traditional label by allowing the representation of not only labels which travel in-band with associated data packets, but also labels which identify time-slots, wavelengths, or space division multiplexed positions. For example, the Generalized Label may carry a label that represents (a) a single fiber in a bundle, (b) a single waveband within fiber, (c) a single wavelength within a waveband (or fiber), or (d) a set of time-slots within a wavelength (or fiber). It may also carry a label that represents a generic MPLS label, a Frame Relay label, or an ATM label $(\mathrm{VCI} / \mathrm{VPI})$.

A Generalized Label does not identify the "class" to which the label belongs. This is implicit in the multiplexing capabilities of the link on which the label is used.

A Generalized Label only carries a single level of label, i.e., it is non-hierarchical. When multiple levels of label (LSPs within LSPS) are required, each LSP must be established separately, see [MPLSHIERARCHY].

Each Generalized Label object/TLV carries a variable length label parameter.

\subsubsection{Required Information}

The information carried in a Generalized Label is:

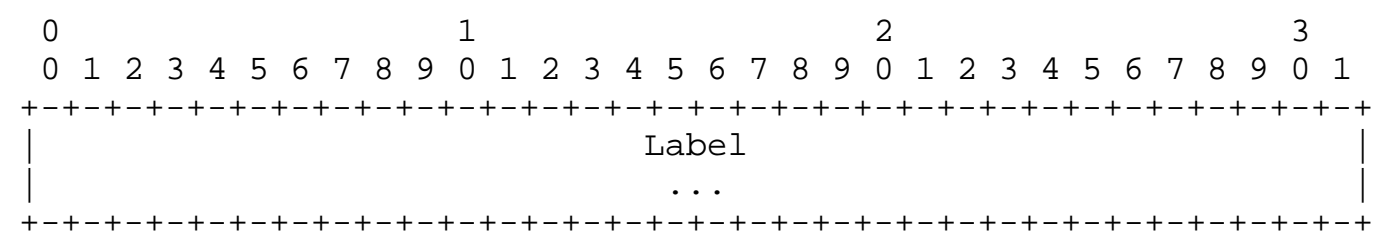

Label: Variable Length

Carries label information. The interpretation of this field depends on the type of the link over which the label is used.

\subsubsection{Port and Wavelength Labels}

Some configurations of fiber switching (FSC) and lambda switching (LSC) use multiple data channels/links controlled by a single control channel. In such cases the label indicates the data channel/link to be used for the LSP. Note that this case is not the same as when [MPLS-BUNDLE] is being used. 
The information carried in a Port and Wavelength label is:

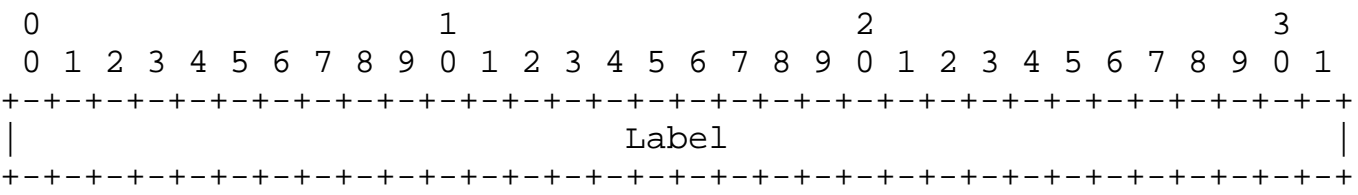

Label: 32 bits

Indicates port/fiber or lambda to be used, from the perspective of the sender of the object/TLV. Values used in this field only have significance between two neighbors, and the receiver may need to convert the received value into a value that has local significance. Values may be configured or dynamically determined using a protocol such as [LMP].

\subsubsection{Other Labels}

Generic MPLS labels and Frame Relay labels are encoded right justified aligned in 32 bits (4 octets). ATM labels are encoded with the VPI right justified in bits 0-15 and the VCI right justified in bits $16-31$.

\subsection{Waveband Switching}

A special case of lambda switching is waveband switching. A waveband represents a set of contiguous wavelengths which can be switched together to a new waveband. For optimization reasons it may be desirable for an optical cross connect to optically switch multiple wavelengths as a unit. This may reduce the distortion on the individual wavelengths and may allow tighter separation of the individual wavelengths. The Waveband Label is defined to support this special case.

Waveband switching naturally introduces another level of label hierarchy and as such the waveband is treated the same way all other upper layer labels are treated.

As far as the MPLS protocols are concerned there is little difference between a waveband label and a wavelength label except that semantically the waveband can be subdivided into wavelengths whereas the wavelength can only be subdivided into time or statistically multiplexed labels. 


\subsubsection{Required information}

Waveband switching uses the same format as the generalized label, see section 3.2 .1 .

In the context of waveband switching, the generalized label has the following format:

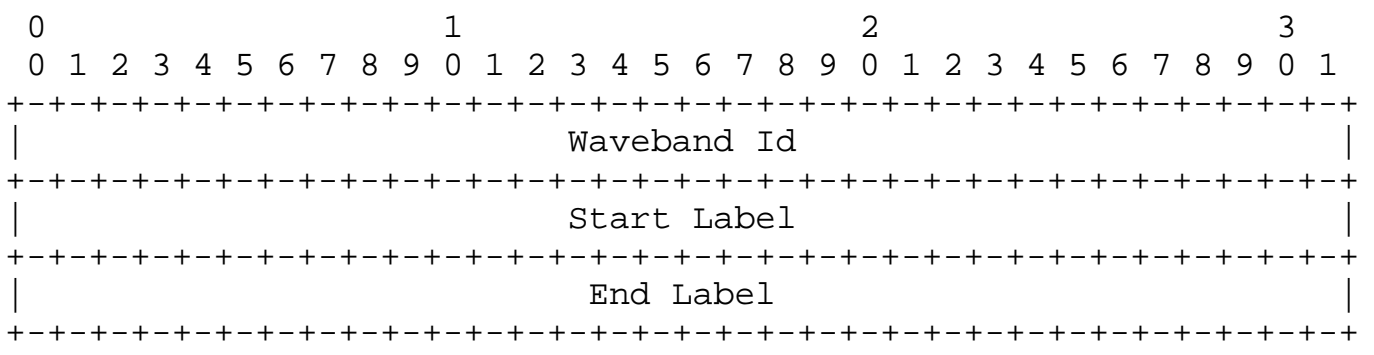

Waveband Id: 32 bits

A waveband identifier. The value is selected by the sender and reused in all subsequent related messages.

Start Label: 32 bits

Indicates the channel identifier of the lowest value wavelength making up the waveband, from the object/TLV sender's

perspective.

End Label: 32 bits

Indicates the channel identifier of the highest value wavelength making up the waveband, from the object/TLV sender's perspective.

Channel identifiers are established either by configuration or by means of a protocol such as LMP [LMP]. They are normally used in the label parameter of the Generalized Label one PSC and LSC.

3.4. Suggested Label

The Suggested Label is used to provide a downstream node with the upstream node's label preference. This permits the upstream node to start configuring its hardware with the proposed label before the label is communicated by the downstream node. Such early configuration is valuable to systems that take non-trivial time to establish a label in hardware. Such early configuration can reduce 
setup latency, and may be important for restoration purposes where alternate LSPs may need to be rapidly established as a result of network failures.

The use of Suggested Label is only an optimization. If a downstream node passes a different label upstream, an upstream LSR reconfigures itself so that it uses the label specified by the downstream node, thereby maintaining the downstream control of a label. Note, the transmission of a suggested label does not imply that the suggested label is available for use. In particular, an ingress node should not transmit data traffic on a suggested label until the downstream node passes a label upstream.

The information carried in a suggested label is identical to a generalized label. Note, values used in the label field of a suggested label are from the object/TLV sender's perspective.

\subsection{Label Set}

The Label set is used to limit the label choices of a downstream node to a set of acceptable labels. This limitation applies on a per hop basis.

We describe four cases where a Label set is useful in the optical domain. The first case is where the end equipment is only capable of transmitting on a small specific set of wavelengths/bands. The second case is where there is a sequence of interfaces which cannot support wavelength conversion (CI-incapable) and require the same wavelength be used end-to-end over a sequence of hops, or even an entire path. The third case is where it is desirable to limit the amount of wavelength conversion being performed to reduce the distortion on the optical signals. The last case is where two ends of a link support different sets of wavelengths.

Label set is used to restrict label ranges that may be used for a particular LSP between two peers. The receiver of a Label set must restrict its choice of labels to one which is in the Label Set. Much like a label, a Label set may be present across multiple hops. In this case each node generates its own outgoing Label set, possibly based on the incoming Label set and the node's hardware capabilities. This case is expected to be the norm for nodes with conversion incapable (CI-incapable) interfaces.

The use of Label set is optional, if not present, all labels from the valid label range may be used. Conceptually the absence of a Label Set implies a Label set whose value is $\{U\}$, the set of all valid labels. 


\subsubsection{Required Information}

A label set is composed of one or more Label_set objects/TLVs. Each object/TLV contains one or more elements of the Label Set. Each element is referred to as a subchannel identifier and has the same format as a generalized label.

The information carried in a Label_set is:

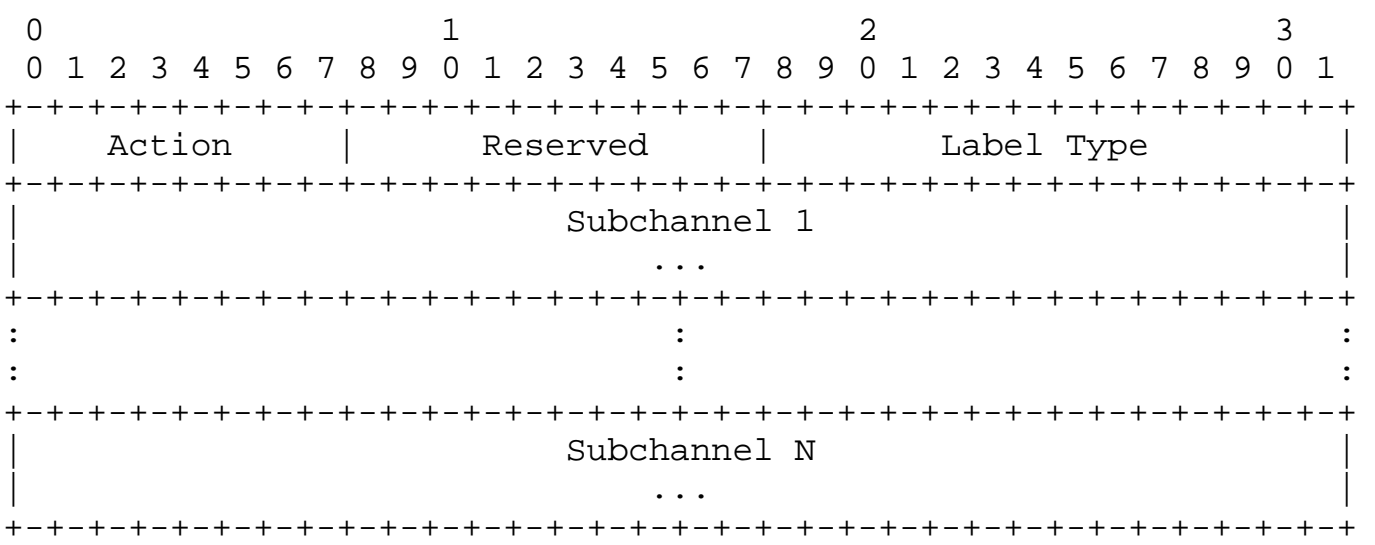

Action: 8 bits

0 - Inclusive List

Indicates that the object/TLV contains one or more subchannel elements that are included in the Label set.

1 - Exclusive List

Indicates that the object/TLV contains one or more subchannel elements that are excluded from the Label set.

2 - Inclusive Range

Indicates that the object/TLV contains a range of labels. The object/TLV contains two subchannel elements. The first element indicates the start of the range. The second element indicates the end of the range. A value of zero indicates that there is no bound on the corresponding portion of the range. 


\section{3 - Exclusive Range}

Indicates that the object/TLV contains a range of labels that are excluded from the Label set. The object/TLV contains two subchannel elements. The first element indicates the start of the range. The second element indicates the end of the range. A value of zero indicates that there is no bound on the corresponding portion of the range.

Reserved: 10 bits

This field is reserved. It MUST be set to zero on transmission and MUST be ignored on receipt.

Label Type: 14 bits

Indicates the type and format of the labels carried in the object/TLV. Values are signaling protocol specific.

Subchannel:

The subchannel represents the label (wavelength, fiber... ) which is eligible for allocation. This field has the same format as described for labels under section 3.2 .

Note that subchannel to local channel identifiers (e.g., wavelength) mappings are a local matter.

\section{Bidirectional LSPS}

This section defines direct support of bidirectional LSPs. Support is defined for LSPs that have the same traffic engineering requirements including fate sharing, protection and restoration, LSRS, and resource requirements (e.g., latency and jitter) in each direction. In the remainder of this section, the term "initiator" is used to refer to a node that starts the establishment of an LSP and the term "terminator" is used to refer to the node that is the target of the LSP. Note that for bidirectional LSPs, there is only one "initiator" and one "terminator".

Normally to establish a bidirectional LSP when using [RFC3209] or [RFC3212] two unidirectional paths must be independently established. This approach has the following disadvantages:

* The latency to establish the bidirectional LSP is equal to one round trip signaling time plus one initiator-terminator signaling transit delay. This not only extends the setup latency for successful LSP establishment, but it extends the worst-case 
latency for discovering an unsuccessful LSP to as much as two times the initiator-terminator transit delay. These delays are particularly significant for LSPs that are established for restoration purposes.

* The control overhead is twice that of a unidirectional LSP. This is because separate control messages (e.g., Path and Resv) must be generated for both segments of the bidirectional LSP.

* Because the resources are established in separate segments, route selection is complicated. There is also additional potential race for conditions in assignment of resources, which decreases the overall probability of successfully establishing the bidirectional connection.

* It is more difficult to provide a clean interface for SONET/SDH equipment that may rely on bidirectional hop-by-hop paths for protection switching.

* Bidirectional optical LSPs (or lightpaths) are seen as a requirement for many optical networking service providers.

With bidirectional LSPs both the downstream and upstream data paths, i.e., from initiator to terminator and terminator to initiator, they are established using a single set of signaling messages. This reduces the setup latency to essentially one initiator-terminator round trip time plus processing time, and limits the control overhead to the same number of messages as a unidirectional LSP.

\subsection{Required Information}

For bidirectional LSPs, two labels must be allocated. Bidirectional LSP setup is indicated by the presence of an Upstream Label object/TLV in the appropriate signaling message. An Upstream Label has the same format as the generalized label, see section 3.2.

\subsection{Contention Resolution}

Contention for labels may occur between two bidirectional LSP setup requests traveling in opposite directions. This contention occurs when both sides allocate the same resources (labels) at effectively the same time. If there is no restriction on the labels that can be used for bidirectional LSPs and if there are alternate resources, then both nodes will pass different labels upstream and there is no contention. However, if there is a restriction on the labels that can be used for the bidirectional LSPs (for example, if they must be physically coupled on a single I/O card), or if there are no more resources available, then the contention must be resolved by other 
means. To resolve contention, the node with the higher node ID will win the contention and it MUST issue a PathErr/NOTIFICATION message with a "Routing problem/Label allocation failure" indication. Upon receipt of such an error, the node SHOULD try to allocate a different Upstream label (and a different Suggested Label if used) to the bidirectional path. However, if no other resources are available, the node must proceed with standard error handling.

To reduce the probability of contention, one may impose a policy that the node with the lower ID never suggests a label in the downstream direction and always accepts a suggested Label from an upstream node with a higher ID. Furthermore, since the labels may be exchanged using LMP, an alternative local policy could further be imposed such that (with respect to the higher numbered node's label set) the higher numbered node could allocate labels from the high end of the label range while the lower numbered node allocates labels from the low end of the label range. This mechanism would augment any close packing algorithms that may be used for bandwidth (or wavelength) optimization. One special case that should be noted when using RSVP and supporting this approach is that the neighbor's node ID might not be known when sending an initial Path message. When this case occurs, a node should suggest a label chosen at random from the available label space.

An example of contention between two nodes (PXC 1 and PXC 2) is shown in Figure 1. In this example PXC 1 assigns an Upstream Label for the channel corresponding to local BCId=2 (local BCId=7 on PXC 2) and sends a Suggested Label for the channel corresponding to local BCId=1 (local BCId=6 on PXC 2). Simultaneously, PXC 2 assigns an Upstream Label for the channel corresponding to its local BCId=6 (local BCId=1 on PXC 1) and sends a Suggested Label for the channel corresponding to its local $\mathrm{BCId}=7$ (local $\mathrm{BCId}=2$ on $\mathrm{PXC} 1$ ). If there is no restriction on the labels that can be used for bidirectional LSPs and if there are alternate resources available, then both PXC 1 and PXC 2 will pass different labels upstream and the contention is resolved naturally (see Fig. 2). However, if there is a restriction on the labels that can be used for bidirectional LSPs (for example, if they must be physically coupled on a single I/O card), then the contention must be resolved using the node ID (see Fig. 3). 


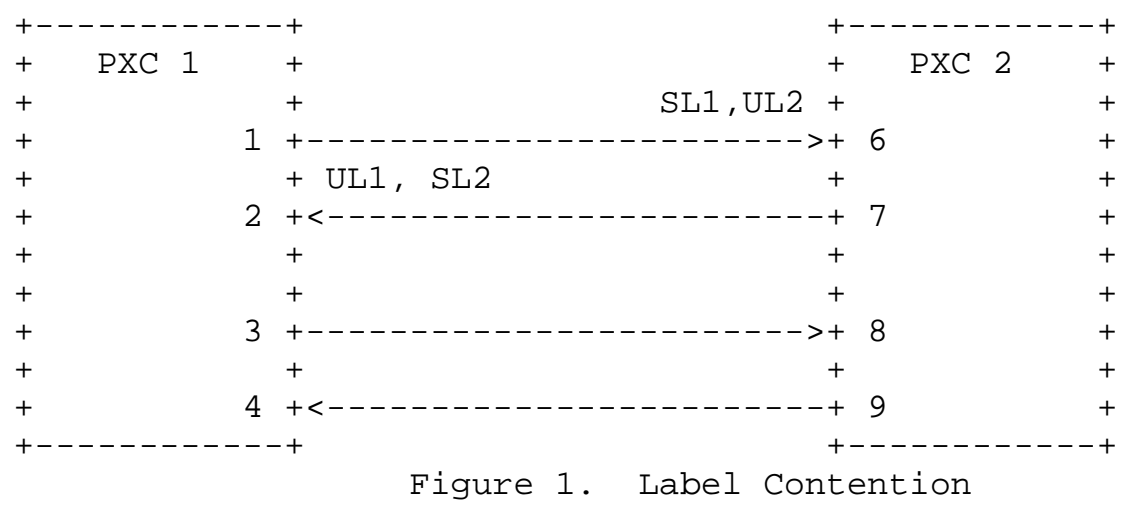

In this example, PXC 1 assigns an Upstream Label using BCId=2 (BCId=7 on PXC 2) and a suggested Label using BCId=1 (BCId=6 on PXC 2). Simultaneously, PXC 2 assigns an Upstream Label using BCId=6 (BCId=1 on PXC 1) and a Suggested Label using BCId=7 (BCId=2 on PXC 1).

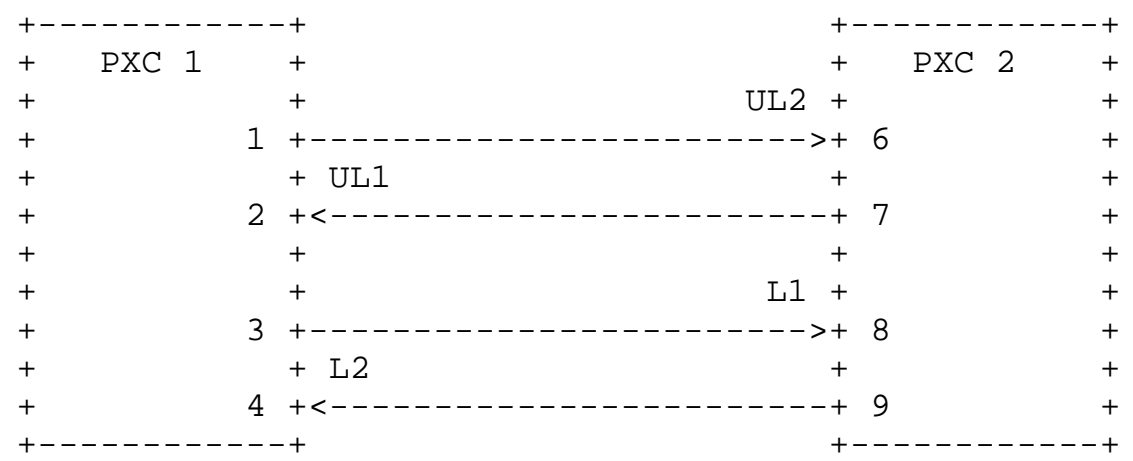

Figure 2. Label Contention Resolution without resource restrictions 
In this example, there is no restriction on the labels that can be used by the bidirectional connection and there is no contention.

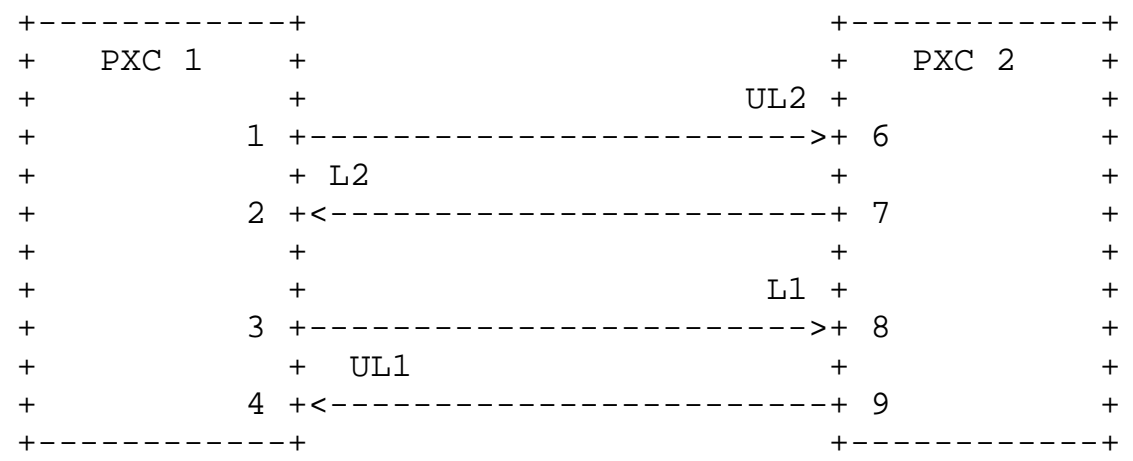

Figure 3. Label Contention Resolution with resource restrictions

In this example, labels 1,2 and 3,4 on PXC 1 (labels 6,7 and 8,9 on PXC 2, respectively) must be used by the same bidirectional connection. Since PXC 2 has a higher node ID, it wins the contention and PXC 1 must use a different set of labels.

5. Notification on Label Error

There are cases in traditional MPLS and in GMPLS that result in an error message containing an "Unacceptable label value" indication, see [RFC3209], [RFC3472] and [RFC3473]. When these cases occur, it can be useful for the node generating the error message to indicate which labels would be acceptable. To cover this case, GMPLS introduces the ability to convey such information via the "Acceptable Label Set". An Acceptable Label set is carried in appropriate protocol specific error messages, see [RFC3472] and [RFC3473].

The format of an Acceptable Label set is identical to a Label Set, see section 3.5.1.

6. Explicit Label Control

In traditional MPLS, the interfaces used by an LSP may be controlled via an explicit route, i.e., ERO or ER-Hop. This enables the inclusion of a particular node/interface, and the termination of an LSP on a particular outgoing interface of the egress LSR. Where the interface may be numbered or unnumbered, see [MPLS-UNNUM] .

There are cases where the existing explicit route semantics do not provide enough information to control the LSP to the degree desired. This occurs in the case when the LSP initiator wishes to select a 
label used on a link. Specifically, the problem is that ERO and ERHop do not support explicit label sub-objects. An example case where such a mechanism is desirable is where there are two LSPs to be "spliced" together, i.e., where the tail of the first LSP would be "spliced" into the head of the second LSP. This last case is more likely to be used in the non-PSC classes of links.

To cover this case, the Label ERO subobject / ER Hop is introduced.

6.1. Required Information

The Label Explicit and Record Routes contains:

$\mathrm{L}: 1$ bit

This bit must be set to 0 .

$\mathrm{U}: 1$ bit

This bit indicates the direction of the label. It is 0 for the downstream label. It is set to 1 for the upstream label and is only used on bidirectional LSPs.

Label: Variable

This field identifies the label to be used. The format of this field is identical to the one used by the Label field in Generalized Label, see Section 3.2.1.

Placement and ordering of these parameters are signaling protocol specific.

7. Protection Information

Protection Information is carried in a new object/TLV. It is used to indicate link related protection attributes of a requested LSP. The use of Protection Information for a particular LSP is optional. Protection Information currently indicates the link protection type desired for the LSP. If a particular protection type, i.e., 1+1, or $1: N$, is requested, then a connection request is processed only if the desired protection type can be honored. Note that the protection capabilities of a link may be advertised in routing, see [GMPLS-RTG] . Path computation algorithms may take this information into account when computing paths for setting up LSPS.

Protection Information also indicates if the LSP is a primary or secondary LSP. A secondary LSP is a backup to a primary LSP. The resources of a secondary LSP are not used until the primary LSP 
fails. The resources allocated for a secondary LSP MAY be used by other LSPs until the primary LSP fails over to the secondary LSP. At that point, any LSP that is using the resources for the secondary LSP MUST be preempted.

7.1. Required Information

The following information is carried in Protection Information:

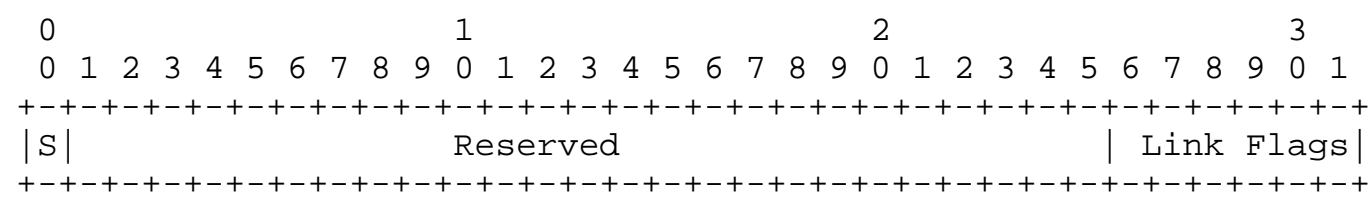

Secondary $(S): 1$ bit

When set, indicates that the requested LSP is a secondary LSP.

Reserved: 25 bits

This field is reserved. It MUST be set to zero on transmission and MUST be ignored on receipt. These bits SHOULD be pass through unmodified by transit nodes.

Link Flags: 6 bits

Indicates desired link protection type. As previously mentioned, protection capabilities of a link may be advertised in routing. A value of 0 implies that any, including no, link protection may be used. More than one bit may be set to indicate when multiple protection types are acceptable. When multiple bits are set and multiple protection types are available, the choice of protection type is a local (policy) decision.

The following flags are defined:

$0 \times 20 \quad$ Enhanced

Indicates that a protection scheme that is more reliable than Dedicated $1+1$ should be used, e.g., 4 fiber BLSR/MS-SPRING. 


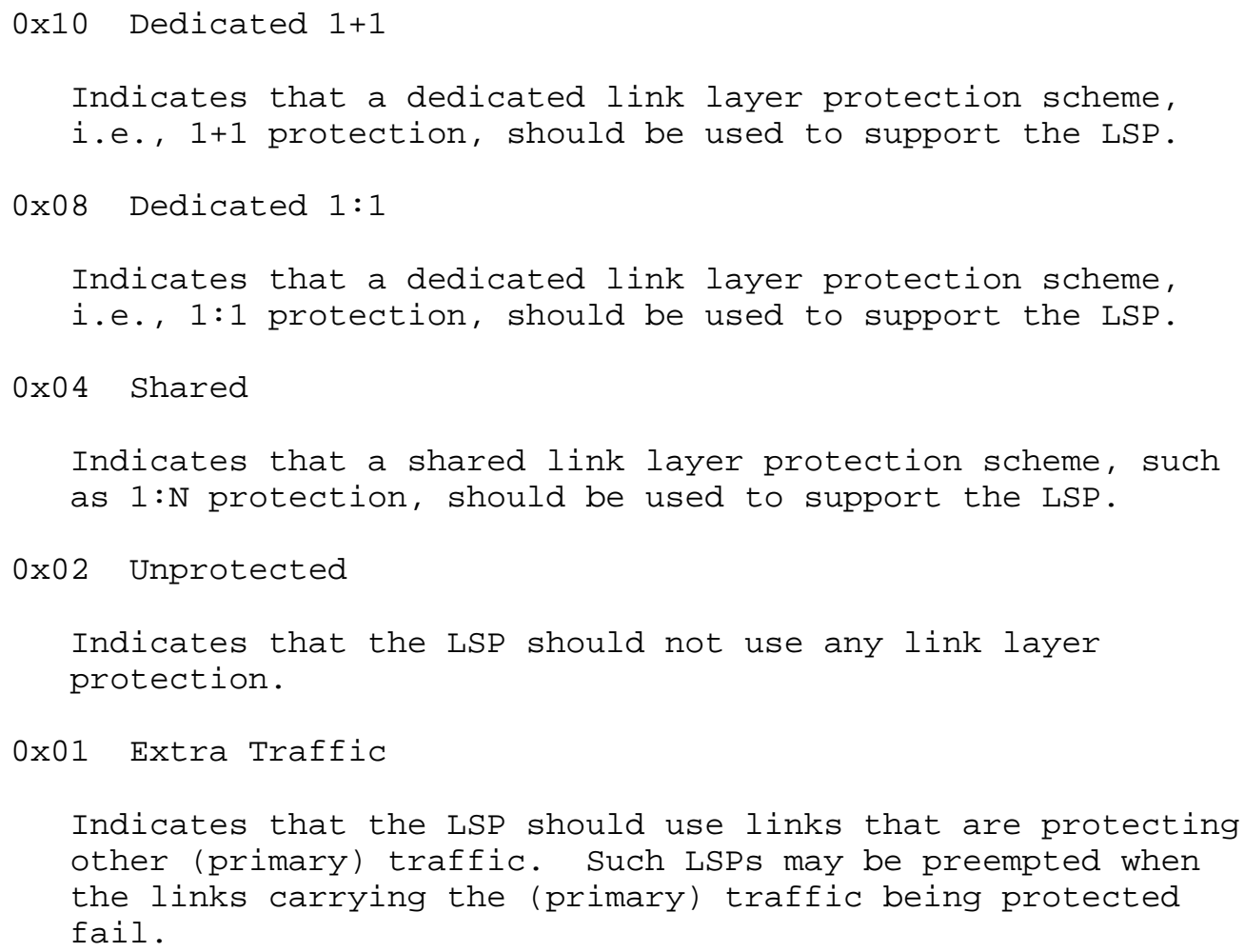

8. Administrative Status Information

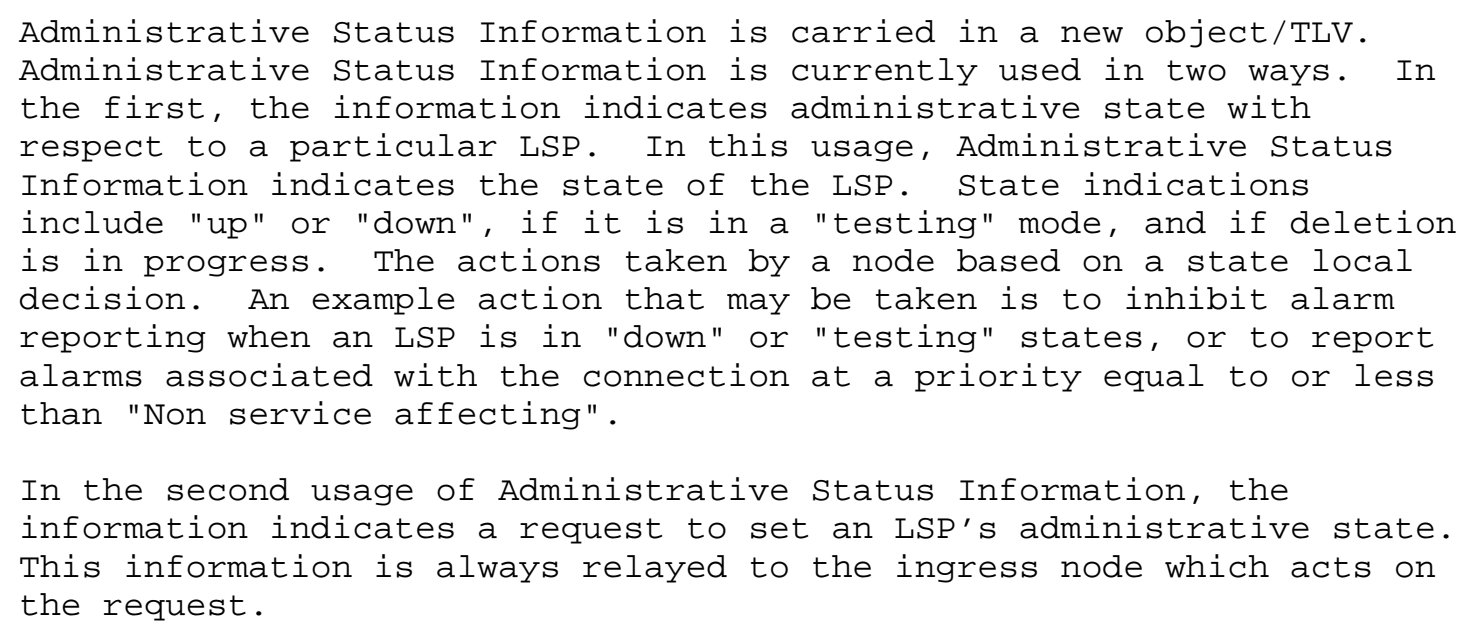


The different usages are distinguished in a protocol specific fashion. See [RFC3473] and [RFC3472] for details. The use of Administrative status Information for a particular LSP is optional.

\subsection{Required Information}

The following information is carried in Administrative status Information:

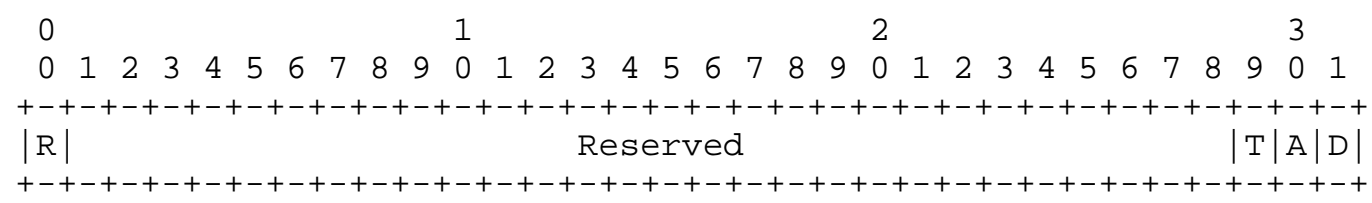

Reflect (R): 1 bit

When set, indicates that the edge node SHOULD reflect the object/TLV back in the appropriate message. This bit MUST NOT be set in state change request, i.e., Notify, messages.

Reserved: 28 bits

This field is reserved. It MUST be set to zero on transmission and MUST be ignored on receipt. These bits SHOULD be pass through unmodified by transit nodes.

Testing $(\mathrm{T}): 1$ bit

When set, indicates that the local actions related to the "testing" mode should be taken.

Administratively down (A) : 1 bit

When set, indicates that the local actions related to the "administratively down" state should be taken.

Deletion in progress (D): 1 bit

When set, indicates that that the local actions related to LSP teardown should be taken. Edge nodes may use this flag to control connection teardown. 


\section{Control Channel Separation}

The concept of a control channel being different than a data channel being signaled was introduced to MPLS in connection with link bundling, see [MPLS-BUNDLE]. In GMPLS, the separation of control and data channel may be due to any number of factors. (Including bundling and other cases such as data channels that cannot carry inband control information.) This section will cover the two critical related issues: the identification of data channels in signaling and handling of control channel failures that don't impact data channels.

\subsection{Interface Identification}

In traditional MPLS there is an implicit one-to-one association of a control channel to a data channel. When such an association is present, no additional or special information is required to associate a particular LSP setup transaction with a particular data channel. (It is implicit in the control channel over which the signaling messages are sent.)

In cases where there is not an explicit one-to-one association of control channels to data channels it is necessary to convey additional information in signaling to identify the particular data channel being controlled. GMPLS supports explicit data channel identification by providing interface identification information. GMPLS allows the use of a number of interface identification schemes including IPV4 or IPV6 addresses, interface indexes (see [MPLSUNNUM]) and component interfaces (established via configuration or a protocol such as [LMP]). In all cases the choice of the data interface is indicated by the upstream node using addresses and identifiers used by the upstream node.

9.1.1. Required Information

The following information is carried in Interface_ID:

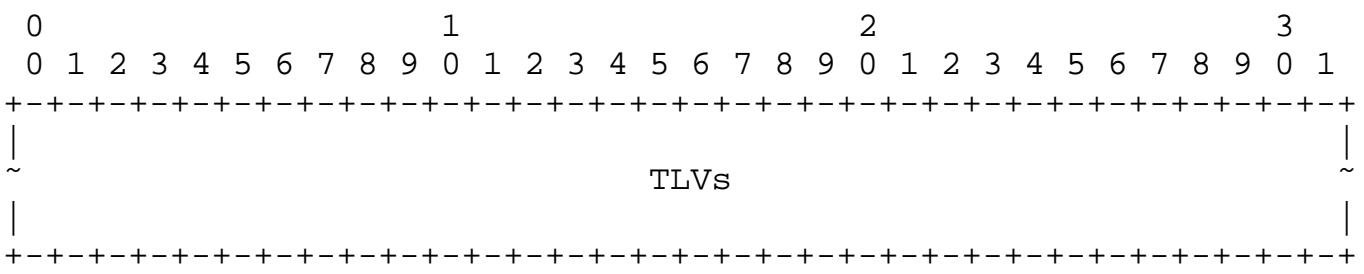


Where each TLV has the following format:

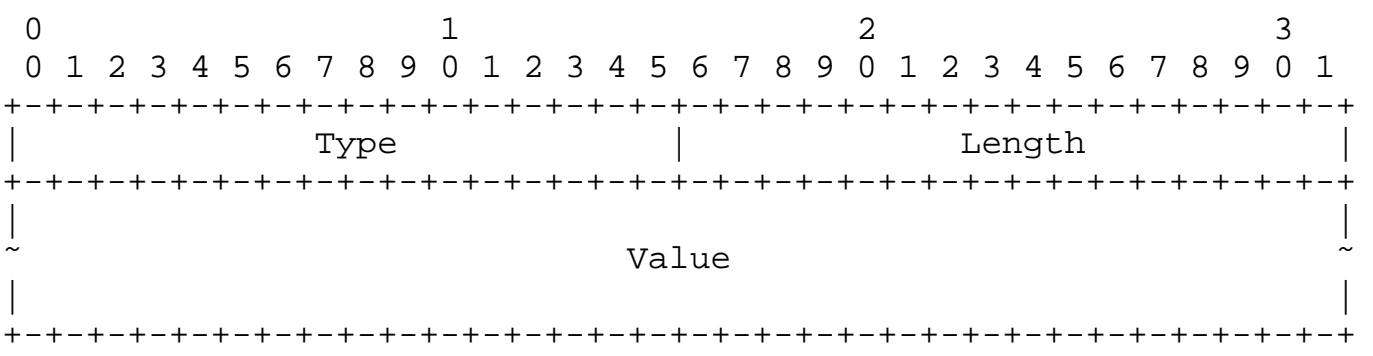

Length: 16 bits

Indicates the total length of the TLV, i.e., 4 + the length of the value field in octets. A value field whose length is not a multiple of four MUST be zero-padded so that the TLV is fouroctet aligned.

Type: 16 bits

Indicates type of interface being identified. Defined values are:

Type Length Format Description

$\begin{array}{lrlll}1 & 8 & \text { IPv4 Addr. IPv4 } & \\ 2 & 20 & \text { IPv6 Addr. IPv6 } & \\ 3 & 12 & \text { See below } & \text { IF_INDEX } & \text { (Interface Index) } \\ 4 & 12 & \text { See below } & \text { COMPONENT_IF_DOWNSTREAM } & \text { (Component interface) } \\ 5 & 12 & \text { See below } & \text { COMPONENT_IF_UPSTREAM } & \text { (Component interface) }\end{array}$

For types 3, 4 and 5 the Value field has the format:

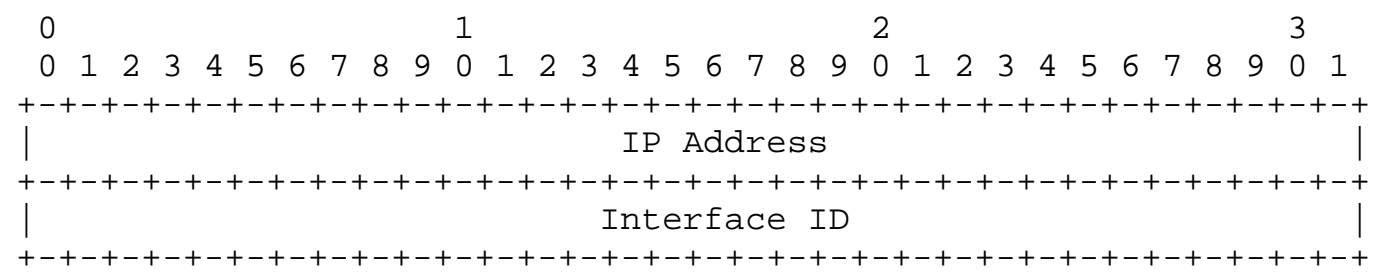

IP Address: 32 bits

The IP address field may carry either an IP address of a link or an IP address associated with the router, where associated address is the value carried in a router address TLV of routing. 


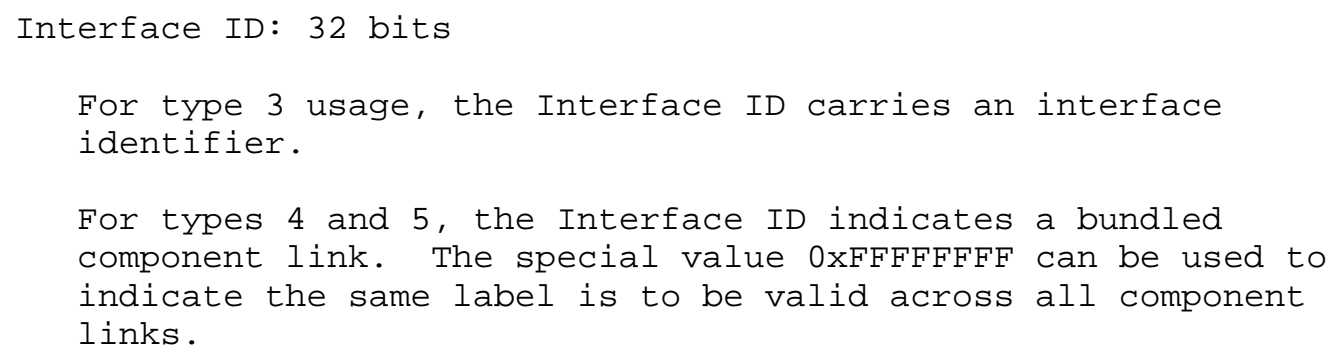

\subsection{Fault Handling}

There are two new faults that must be handled when the control channel is independent of the data channel. In the first, there is a link or other type of failure that limits the ability of neighboring nodes to pass control messages. In this situation, neighboring nodes are unable to exchange control messages for a period of time. Once communication is restored the underlying signaling protocol must indicate that the nodes have maintained their state through the failure. The signaling protocol must also ensure that any state changes that were instantiated during the failure are synchronized between the nodes.

In the second, a node's control plane fails and then restarts and losses most of its state information. In this case, both upstream and downstream nodes must synchronize their state information with the restarted node. In order for any resynchronization to occur the node undergoing the restart will need to preserve some information, such as its mappings of incoming to outgoing labels.

Both cases are addressed in protocol specific fashions, see [RFC3473] and [RFC3472].

Note that these cases only apply when there are mechanisms to detect data channel failures independent of control channel failures.

10. Acknowledgments

This document is the work of numerous authors and consists of a composition of a number of previous documents in this area.

Valuable comments and input were received from a number of people, including Igor Bryskin, Adrian Farrel, Ben Mack-Crane, Dimitri Papadimitriou, Fong Liaw and Juergen Heiles. Some sections of this document are based on text proposed by Fong Liaw. 


\section{Security Considerations}

This document introduce no new security considerations to either [RFC3212] or [RFC3209]. The security considerations mentioned in [RFC3212] or [RFC3209] apply to the respective protocol specific forms of GMPLS, see [RFC3473] and [RFC3472].

12. IANA Considerations

The IANA will administer assignment of new values for namespaces defined in this document. This section uses the terminology of $B C P$ 26 "Guidelines for Writing an IANA Considerations Section in RFCs" [BCP 26].

This document defines the following namespaces:

- LSP Encoding Type: 8 bits

o Switching Type: 8 bits

- Generalized PID (G-PID) : 16 bits

o Action: 8 bits

o Interface_ID Type: 16 bits

All future assignments should be allocated through IETF Consensus action or documented in a specification.

LSP Encoding Type - valid value range is 1-255. This document defines values $1-11$.

Switching Type - valid value range is 1-255. This document defines values $1-4,100,150$ and 200 .

Generalized PID (G-PID) - valid value range is 0-1500. This document defines values $0-46$.

Action - valid value range is 0-255. This document defines values $0-3$.

Interface_ID Type - valid value range is 1-65535. This document defines values 1-5. 


\section{Intellectual Property Considerations}

This section is taken from Section 10.4 of [RFC2026].

The IETF takes no position regarding the validity or scope of any intellectual property or other rights that might be claimed to pertain to the implementation or use of the technology described in this document or the extent to which any license under such rights might or might not be available; neither does it represent that it has made any effort to identify any such rights. Information on the IETF's procedures with respect to rights in standards-track and standards-related documentation can be found in BCP-11. Copies of claims of rights made available for publication and any assurances of licenses to be made available, or the result of an attempt made to obtain a general license or permission for the use of such proprietary rights by implementors or users of this specification can be obtained from the IETF secretariat.

The IETF invites any interested party to bring to its attention any copyrights, patents or patent applications, or other proprietary rights which may cover technology that may be required to practice this standard. Please address the information to the IETF Executive Director.

14. References

14.1. Normative References

[RFC2119] Bradner, S., "Key words for use in RFCs to Indicate Requirement Levels," BCP 14, RFC 2119, March 1997.

[RFC3036] Andersson, L., Doolan, P., Feldman, N., Fredette, A. and B. Thomas, "LDP Specification", RFC 3036, January 2001 .

[RFC3209] Awduche, D., Berger, L., Gan, D., Li, T., Srinivasan, V. and G. Swallow, "RSVP-TE: Extensions to RSVP for LSP Tunnels", RFC 3209, December 2001.

[RFC3212]

Jamoussi, B., Andersson, L., Callon, R., Dantu, R., Wu, L., Doolan, P., Worster, T., Feldman, N., Fredette, A., Girish, M., Gray, E., Heinanen, J., Kilty, T. and A. Malis, "Constraint-Based LSP Setup using LDP", RFC 3212, January 2002. 
[RFC3472]

Ashwood-Smith, P. and L. Berger, Editors, "Generalized Multi-Protocol Label Switching (GMPLS) Signaling - Constraint-based Routed Label Distribution Protocol (CR-LDP) Extensions", RFC 3472, January 2003.

[RFC3473]

Berger, L., Editor "Generalized Multi-Protocol Label Switching (GMPLS) Signaling - Resource ReserVation Protocol-Traffic Engineering (RSVP-TE) Extensions", RFC 3473, January 2003.

14.2. Informative References

[GMPLS-RTG] Kompella, K., et al., "Routing Extensions in Support of Generalized MPLS", Work in Progress.

[GMPLS-SONET] Ashwood-Smith, P., et al., "GMPLS - SONET / SDH Specifics", Work in Progress.

[LMP] Lang, et al., "Link Management Protocol", Work in Progress.

[MPLS-BUNDLE] Kompella, K., Rekhter, Y. and L. Berger, "Link Bundling in MPLS Traffic Engineering", Work in Progress.

[MPLS-HIERARCHY] Kompella, K. and Y. Rekhter, "LSP Hierarchy with MPLS TE", Work in Progress.

[RFC2026] Bradner, S., "The Internet Standards Process -Revision 3," BCP 9, RFC 2026, October 1996.

[RFC2434] Narten, T. and H. Alvestrand, "Guidelines for Writing an IANA Considerations Section in RFCs", BCP 26, RFC 2434, October 1998.

[RFC3031] Rosen, E., Viswanathan, A. and R. Callon, "Multiprotocol label switching Architecture", RFC 3031, January 2001. 
15. Contributors

Peter Ashwood-Smith

Nortel Networks Corp.

P.O. Box 3511 Station C,

Ottawa, ON K1Y $4 \mathrm{H} 7$

Canada

Phone: +16137634534

EMail: petera@nortelnetworks.com

Ayan Banerjee

Calient Networks

5853 Rue Ferrari

San Jose, CA 95138

Phone: +1408 972-3645

EMail: abanerjeedcalient.net

Lou Berger

Movaz Networks, Inc.

7926 Jones Branch Drive

Suite 615

McLean VA, 22102

Phone: +1703 847-1801

EMail: lberger@movaz.com

Greg Bernstein

EMail: gregbegrotto-networking.com

John Drake

Calient Networks

5853 Rue Ferrari

San Jose, CA 95138

Phone: +1408 9723720

EMail: jdrake@calient.net 


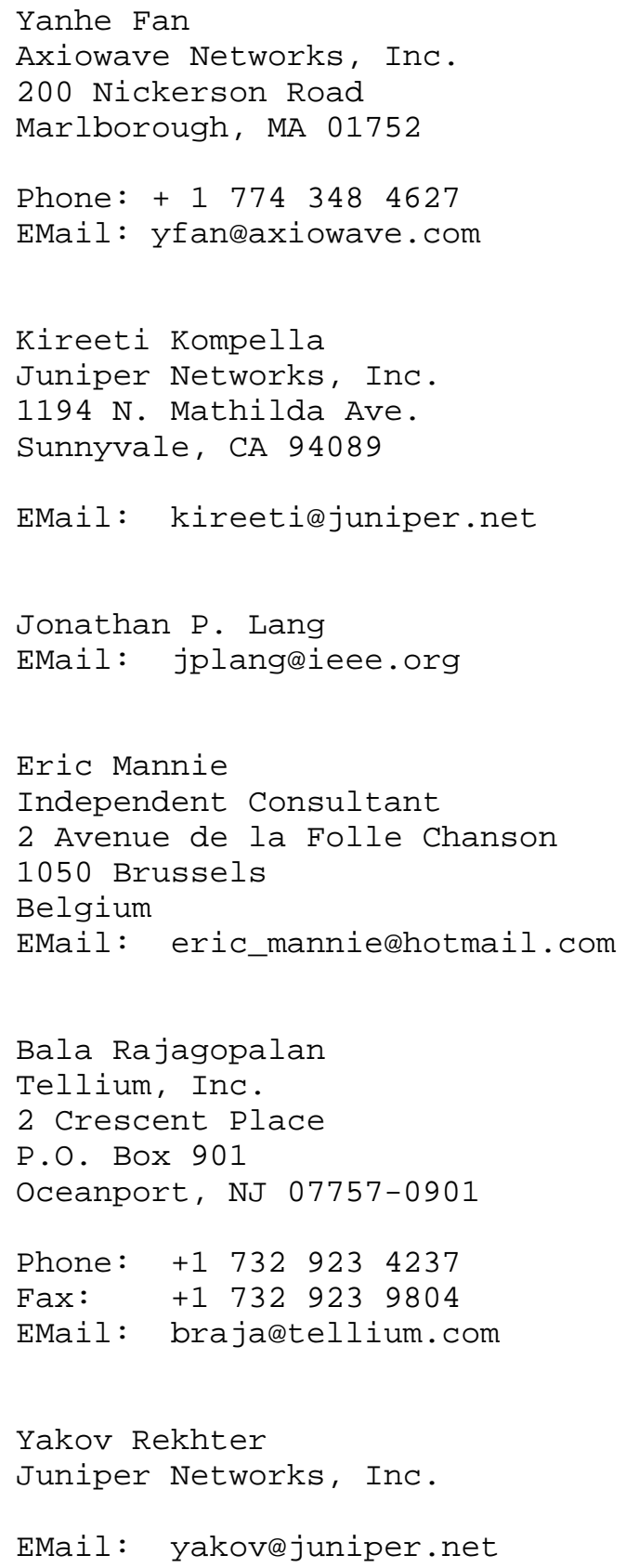




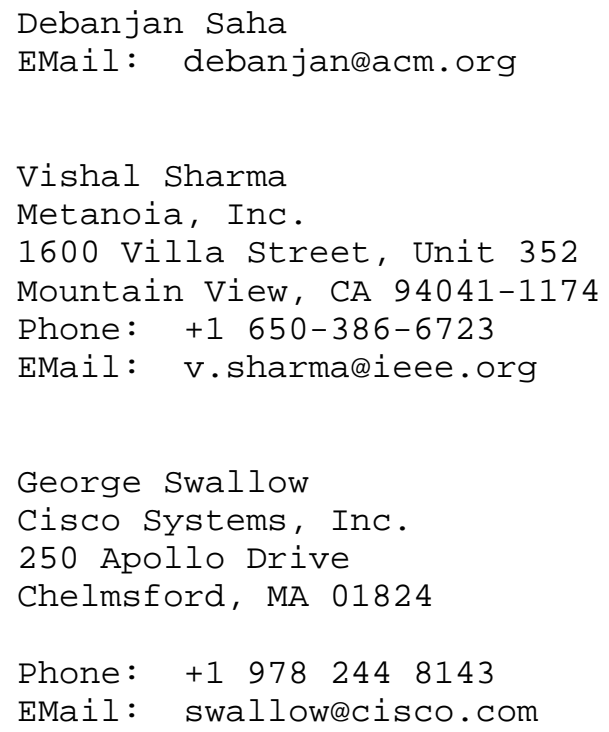


17. Full Copyright statement

Copyright (C) The Internet Society (2003). All Rights Reserved.

This document and translations of it may be copied and furnished to others, and derivative works that comment on or otherwise explain it or assist in its implementation may be prepared, copied, published and distributed, in whole or in part, without restriction of any kind, provided that the above copyright notice and this paragraph are included on all such copies and derivative works. However, this document itself may not be modified in any way, such as by removing the copyright notice or references to the Internet society or other Internet organizations, except as needed for the purpose of developing Internet standards in which case the procedures for copyrights defined in the Internet Standards process must be followed, or as required to translate it into languages other than English.

The limited permissions granted above are perpetual and will not be revoked by the Internet society or its successors or assigns.

This document and the information contained herein is provided on an "AS IS" basis and THE INTERNET SOCIETY AND THE INTERNET ENGINEERING TASK FORCE DISCLAIMS ALL WARRANTIES, EXPRESS OR IMPLIED, INCLUDING BUT NOT LIMITED TO ANY WARRANTY THAT THE USE OF THE INFORMATION HEREIN WILL NOT INFRINGE ANY RIGHTS OR ANY IMPLIED WARRANTIES OF MERCHANTABILITY OR FITNESS FOR A PARTICULAR PURPOSE.

Acknowledgement

Funding for the RFC Editor function is currently provided by the Internet society. 